\title{
Retorno ao trabalho após revascularização do miocárdio*
}

\author{
Return to work after myocardical revascularization
}

Olímpio J.N.V. Bittar**

\begin{abstract}
BITTAR, O.J.N.V. Retorno ao trabalho após revascularização do miocárdio. Rev. Saúde Pública, 27: 195-203,1993. Seiscentos e setenta e dois pacientes, classificados em 4 estratos de 168 cada um, foram avaliados no quarto mês após revascularização miocárdica, por cirurgia de ponte de safena e/ou implantação de artéria mamária e angioplastia transluminal coronária com o intuito de verificar se retomaram ou não ao trabalho, e em que condiçōes o fizeram. Os quatro estratos foram compostos segundo a ocupação/qualificação da seguinte forma: I- executivos; IIprofissionais de nível universitário; III- técnicos de nível médio; IV-profissionais não ou semi qualificados. Objetivou-se verificar como as variáveis demográficas, educacionais, ocupacionais, procedimentos e suporte pós-operatório interferiram no retorno ao trabalho. Os resultados mostraram que o não-retorno aconteceu, em média, em $20,8 \%$ dos casos. Em cada um dos estratos, a proporção de não-retomo foi a seguinte: $I=11,9 \% ; \Pi=15,5 \% ; \quad I I=26,2 \%$ e $I V=29,8 \%$. Verificou-se naqueles submetidos à angioplastia que a proporção de não-retorno foi significativamente menor.
\end{abstract}

Descritores: Revascularização miocárdica, reabilitação. Qualidade de vida. Trabalho.

\section{1 - Introdução}

$\mathrm{Na}$ vigésima conferência de Bethesda (Maryland - EUA) realizada pelo "American College of Cardiology" e pela "Association of Life Insurance Medical Directors of America", em outubro de 1988, cujo tema foi "Insurability and Employability of the Patient With Ischemic Heart Disease", entre os tópicos discutidos estavam os fatores que influenciam o retorno ao trabalho de pacientes portadores de doença isquêmica do coração.

Segundo DeBusk ${ }^{9}$, os objetivos da conferência eram os de definir políticas de seguridade e de emprego, que maximizassem o potencial ocupacional do paciente com doença isquêmica do coração, e ao mesmo tempo protegessem a segurança pública, de

\footnotetext{
* Pesquisa subvencionada pela CAPES. Parte de Tese de Doutorado, sob o mesmo título, apresentada ao Departamento de Medicina Social da Faculdade de Medicina de Ribeirão Preto da Universidade de São Paulo, em agosto de 1992

* Instituto Dante Pazzanese de Cardiologia - São Paulo, SP - Brasil
}

Separatas/Reprints: O.J.N.V. Bittar - Av. Dr. Dante Pazzanese, 500 - Ibirapuera - 04012-900 - São Paulo, SP - Brasil

Edição subvencionada pela FAPESP. Processo Medicina 93/ 0208-5. modo a permitir ou estimular a volta ao trabalho de indivíduos em atividades ligadas diretamente ao público (como é o caso dos transportes que tem entre os profissionais, pilotos de aeronaves e motoristas de veículos coletivos).

Guillette e col. ${ }^{12}$ incluíram os seguintes fatores que exercem a citada influência: gravidade da doença cardiaca; sexo; classe social; tipo de trabalho; distúrbios emocionais; falta de cooperação do empregador; super proteção da família; ausência de programas de reabilitação; mercado de trabalho, desemprego, benefícios sociais; idade; ocupação; educação; renda familiar; residência urbana ou rural; atitude do médico; atraso na avaliação dos pacientes; outros problemas médicos.

Série de estudos iniciada em 1968 e que se estendeu até o final da década passada, concentrando-se a maioria deles na década de 70 , mostra que o não-retorno ao trabalho variou enormemente, em uma faixa de $6 \%$ a $83 \%$. Especificamente a este último percentual foi encontrado por Gundle e col. ${ }^{13}$, porém o tamanho amostral da pesquisa era de 30 pacientes e a percentagem dos que estavam trabalhando antes do procedimento era de apenas $22 \%$, o que segundo os autores teve influência.

Mesmo em país como o Canadá, Vaislic e Passas $^{32}$ encontraram diferenças significativas quando compararam pacientes de duas províncias, alertando que o resultado imediato depende essencialmente de fatores socioeconômicos. 
Danchin e col..$^{5}$ observaram que as discrepâncias entre os estudos pode ser explicada, em parte, pelos diferentes métodos de seleção dos pacientes, pela definição do que é "status" de trabalho e pelo periodo de latencia entre o procedimento e a avaliação. Em seu estudo, não foram incluídas mulheres porque suas ocupaçōes são freqüentemente diferentes das dos homens e seu status de trabalho é mais difícil de ser avaliado.

Outros critérios avaliados por Danchin e col. ${ }^{5}$ foram a idade e a morte logo após o procedimento, sendo que em alguns trabalhos citados por esse autor, os pacientes que chegaram ao óbito foram contados como não tendo retomado.

Outra discrepância observada foi a de que alguns dos autores consultados incluíram nas pesquisas pacientes aposentados e desempregados, sendo que as chances de retorno dos primeiros são menores.

A maioria dos autores acha que a quantidade de pacientes que não retorna ao trabalho poderia ser menor, o que coincide com a experiência de Ro$\mathrm{dis}^{26}$, que é quem apresentou o melhor resultado: $6 \%$ de não-retorno e um aumento de $6 \%$ na força de trabalho pós cirurgia.

David e col. ${ }^{8}$ invocam quatro motivos para explicar as diferenças entre os estudos: 1-tipo diferente de clientela do ponto de vista médico e socioeconômico; 2- diversidade de métodos utilizados para avaliar o retorno ao trabalho; 3 - duração do seguimento dos pacientes; e 4- inclusão de mulheres, de doentes idosos e dos óbitos.

Haskell e col. ${ }^{15}$ vão mais diretamente ao assunto, quando dizem que "alguns pacientes que não se encontram satisfeitos com seu emprego tiram vantagem da oportunidade de aposentar, oferecida pelo infarto ou pela intervenção cirúrgica, mesmo tendo condições seguras de retornar ao trabalho".

O objetivo do presente estudo foi, analisar o retomo ao trabalho de pacientes submetidos à revascularização do miocárdio através de dois procedimentos: a cirurgia de implantação de ponte de safena e/ou artéria mamária e a angioplastia transluminal coronária. A análise abrangerá as condições e fatores que interferem no retorno do paciente ao trabalho, tais como: ocupação, idade, sexo, antecedentes, hábitos, perfil bioquímico, restriçð̃es do empregador, condiçס̃es psicológicas (medo, ansiedade, percepção) e de apoio pósoperatório.

\section{2 - Materlal e Método}

No período compreendido entre janeiro de 1990 e junho de 1991, foram selecionados 672 pacientes atendidos em dois hospitais especializados em doenças cardiovasculares, com tradição nas áreas de assistência médica, ensino e pesquisa, localizados na cidade de São Paulo. A inclusão dos pacientes foi feita com os seguintes critérios: a) pacientes com lesão coronária maior ou igual a $75 \%$ do lumen obstruído, uni ou multiarterial, com indicação de revascularização do miocárdio por ponte de safena e/ou artéria mamária ou angioplastia transluminal coronária; b) pacientes de ambos os sexos, com idade inferior a 65 anos, empregados ou não; c) a admissão dos pacientes foi feita consecutivamente.

Não foram incluídos os pacientes submetidos ao procedimento em condições de emergência, aqueles portadores de outras patologias que seriam corrigidas no momento do procedimento, como aneurismas de ventrículo e de aorta, disfunções valvulares e portadores de marca-passo cardíaco, e os aposentados.

Distribuiu-se a população em quatro estratos ocupacionais, assim compostos:

\section{EI - Estrato Executivo}

Formado por profissionais cuja função na organização não está diretamente, ou necessariamente vinculada à formação acadêmica, sendo outros os requisitos exigidos para a atuação profissional, dedicando-se à tomada de decisōes e/ou à liderança de funcionários. Esta categoria profissional, composta por proprietários, presidentes, diretores e gerentes é conhecida como executiva. Foram agregados a ela, neste trabalho, as altas patentes militares, bem como executivos de empresas públicas.

\section{EII - Estrato Universitário}

Constituído por profissionais cuja função da organização guarda estreita interdependência com a formação acadêmica em nível superior, como suporte técnico a suas decisőes. Foram englobados os profissionais liberais, supervisores de primeira linha, assessoria técnica, analistas e oficiais militares.

\section{EIII - Estrato de técnicos de nível médio}

Este estrato englobou técnicos de nível médio, atuantes na indústria, comércio, agricultura e serviços, como por exemplo, técnicos de laboratório, técnicos de enfermagem, técnicos de manutenção, programadores de computador e técnicos em agrimensura. Foram incluídos também os microempresários que, além da administração geral dos negócios, atuam como técnicos nas áreas de produção e os suboficiais militares. 
EIV - Estrato de profissionais não qualificados ou semiqualificados

Foram incluídos os profissionais que, pela natureza do trabalho, não se exige formação acadêmica. São os ajudantes de um modo geral, auxiliares, atendentes e domésticas, sem escolaridade ou cujo nivel educacional é equivalente ao primeiro grau. As patentes militares de cabo e soldado foram incluídas neste estrato.

A estratificação acima teve como critério para agrupamento das ocupaçð̃es, a natureza do trabalho e o grau de complexidade das tarefas que definem nível hierárquico como também de escolaridade, especialização, habilidades envolvidas, grau de risco e esforço físico.

\section{3 - Resultados e Discussáo}

Dos 672 pacientes, 546 pertenciam ao sexo masculino, sendo que das 126 mulheres, 79,4\% delas concentraram-se no estrato IV, em virtude de exercerem, na sua maioria, exclusivamente atividades no lar (Tabela 1).

Quanto à cor, 93,8\% eram brancos e quanto ao estado civil $87,1 \%$ eram casados ou viviam acompanhados.

Quanto à idade, 93,8\% dos homens e 99,2\% das mulheres encontravam-se entre 40-65 anos (Tabela 1).

O número de cirurgia foi de 374 e o de angioplastias 298 , sendo que as mulheres foram submetidas à angioplastia em maior número, enquanto que os homens, à cirurgia.

Tabela 1. Distribuiçăo por faixa etária, sexo e estrato ocupacional dos pacientes.

\begin{tabular}{|c|c|c|c|c|c|c|c|c|c|c|c|}
\hline $\begin{array}{r}\text { Extrato } \\
\text { Sexo } \\
\text { Faixa etária }\end{array}$ & Masc. ' & Fem. & Masc. & Fem. & Masc. & Fem. & Masc. & Fem. & $\begin{array}{l}\text { Sub } \\
\text { Masc. }\end{array}$ & $\begin{array}{l}\text { total } \\
\text { Fem. }\end{array}$ & Total \\
\hline $\begin{array}{r}0-29 \\
30-39 \\
40-49 \\
50-59 \\
60-65\end{array}$ & $\begin{array}{l}- \\
9 \\
49 \\
82 \\
26\end{array}$ & $\begin{array}{l}- \\
- \\
- \\
-\end{array}$ & $\begin{array}{l}- \\
13 \\
61 \\
61 \\
24\end{array}$ & $\begin{array}{l}- \\
- \\
3 \\
5 \\
1\end{array}$ & $\begin{array}{r}1 \\
6 \\
54 \\
62 \\
30\end{array}$ & $\begin{array}{r}- \\
1 \\
2 \\
10 \\
2\end{array}$ & $\begin{array}{r}\overline{5} \\
22 \\
19 \\
22\end{array}$ & $\begin{array}{r}- \\
19 \\
39 \\
42\end{array}$ & $\begin{array}{r}1 \\
33 \\
186 \\
224 \\
102\end{array}$ & $\begin{array}{r}- \\
26 \\
54 \\
45\end{array}$ & $\begin{array}{r}1 \\
34 \\
212 \\
278 \\
147\end{array}$ \\
\hline Subtotal & 166 & 2 & 159 & 9 & 153 & 15 & 68 & 100 & 546 & 126 & 672 \\
\hline Total & 168 & & 168 & & 168 & & 168 & & 672 & & 672 \\
\hline
\end{tabular}

Para cada estrato, o tamanho mínimo da amostra foi de 168 pacientes, tendo como estimativa do parâmetro "nāo-retorno" igual a $12,5 \%$, erro máximo de amostragem de $5 \%$ e coeficiente de confiança de $95 \%$.

A análise estatística das variáveis quantitativas foi feita usando-se a análise de variância e o teste " $t$ " de Student. Para as qualitativas, a análise foi realizada com o qui-quadrado e o teste exato de Fisher. As decisōes foram tomadas ao nivel de significância de 0,05 . Para as proporçōes foi feito o cálculo do intervalo de confiança da verdadeira proporção (coeficiente de confiança 95\%).

As variáveis selecionadas foram as demográficas, educacionais, ocupacionais, antecedentes, hábitos, perfil bioquímico, perfil psicológico (medo, ansiedade, percepção), de "suporte" pósoperatório, atividades de lazer, de relacionamento social, bem estar físico e mental.

Os pacientes foram entrevistados por uma. única entrevistadora, em duas ocasioes, uma antes do procedimento e outra no quarto mês após o procedimento, visto que, ao final do segundo mês, esses pacientes deveriam reiniciar suas atividades plenamente.
O maior número de cirurgias ocorreu nos estratos I e II.

\section{1 - Näo-Retorno ao Trabalho}

Na Tabela 2 é apresentada a distribuição e proporção de pacientes submetidos à cirurgia e angioplastia que não retornaram ao trabalho, de acordo com os estratos ocupacionais, verificando-se que a proporção de não-retorno é crescente dos estratos I ao IV. Assim, no I não retornaram $11,9 \%$, no II

Tabela 2. Não-retorno ao trabalho original de acordo com o procedimento e estrato ocupacional.

\begin{tabular}{lrrrrrrrrrr}
\hline $\begin{array}{l}\text { Procedi- } \\
\text { mento }\end{array}$ & Angioplastia & \multicolumn{2}{c}{ Cirurgia } & \multicolumn{3}{l}{ Total } \\
\hline Extrato & Ne & N. ret. $\%$ & N & N. ret. $\%$ & N & N. ret. $\%$ \\
\hline I & 56 & 6 & 10,7 & 112 & 14 & 12,5 & 168 & 20 & 11.9 \\
II & 65 & 10 & 15,4 & 103 & 16 & 15,5 & 168 & 26 & 15.5 \\
III & 91 & 15 & 16,5 & 77 & 29 & 37,7 & 168 & 44 & 26.2 \\
IV & 86 & 19 & 22,0 & 82 & 31 & 37,8 & 168 & 50 & 29.8 \\
\hline Total & 298 & 50 & 16,8 & 374 & 90 & 24,1 & 672 & 140 & 20.8 \\
\hline
\end{tabular}


$15,5 \%$, no III $26,2 \%$, e no IV $29,8 \%$, resultando um valor médio de $20,8 \%$ (17,8-23,9\%).

\subsection{1 - Quanto aos estratos ocupacionais}

As proporções de não-retorno são crescentes, do estrato mais alto para o mais baixo, independentemente do procedimento de revascularização. Este fato sugere que a causa importante do nãoretomo está associada ao tipo de estrato, que guarda relação com o nível educacional e com o tipo de ocupação exercida.

Como foi visto na literatura, o nivel educacional guarda uma relação bastante estreita com a ocupação e também com a renda. No presente estudo, como já foi descrito anteriormente, com exceção do estrato II, onde o critério de inclusão exigia nível superior, o nível de escolarização aumentou do estrato IV para o I.

Lidlle e col. ${ }^{20}$ encontraram resultados semethantes analisando o nivel educacional, o que os fez afirmar a importância dessa variável no retorno ao trabalho.

Verificou-se, também, na Tabela 2, que a percentagem de não-retorno no estrato IV é quase três vezes maior que no estrato I, e duas vezes maior que no estrato II, sendo que a do estrato III é ligeiramente inferior ao IV.

$\mathrm{Rimm}^{25}$ mostra que a relação de não-retorno de operários com ou sem habilidade é duas vezes maior do que a de profissionais, administradores, burocratas e vendedores, após cirurgia.

A associação entre o tipo de ocupação, isto é, aquelas que exigem esforço leve e moderado e as que exigem esforço pesado, é relatada por Boulay e col. ${ }^{2}$, sendo nas primeiras encontradas maiores probabilidades de retorno.

A aplicação do teste qui-quadrado entre os estratos I e II não apresentou diferença estatisticamente significante ( $p>0,05)$, o mesmo ocorrendo com os estratos III e IV ( $p>0,05)$. Porém confrontando-se os estratos I e II com os estratos III e IV a diferença estatística é altamente significante $(\mathrm{p}<0,000001)$, o que pode levar à consideração da existência de somente dois estratos, um superior e o outro inferior, no período em que esta pesquisa foi realizada.

\subsection{2 - Quanto ao procedimento}

Analisando a Tabela 2, verifica-se que dos 140 pacientes que não retornaram ao trabalho, 90 eram pacientes cirúrgicos. A aplicação do teste do qui-quadrado, demonstra que existe uma associação entre o não-retorno ao trabalho e o tipo de procedimento $(\mathrm{p}<0,05)$, favorecendo aqueles pacientes submetidos à angioplastia, ou seja, estes têm maior probabilidade de retorno ao trabalho. Entre aqueles que foram submetidos à cirurgia (374), 24,1\% não retornaram enquanto que, dos submetidos à angioplastia (298), 16,8\% não o fizeram.

Esta conclusão já foi encontrada por autores como Holmes e col. ${ }^{17}$ que citam como vantagens o fato de o paciente de angioplastia ter, na maioria dos casos, um único vaso lesado, uma melhor função ventricular, ser mais jovem e ter baixa incidência de infarto e angina.

Holmes e col. ${ }^{16}$, fazendo um estudo comparativo entre pacientes submetidos à angioplastia com sucesso, angioplastia com insucesso seguida de cirurgia e angioplastia com insucesso seguida de tratamento clínico, relatam que a angioplastia, embora menos invasiva e associada com um período curto de convalescença, e menor morbidade, não apresenta vantagens no seu emprego nesses três grupos. Reafirmam porém que o tempo de latência para os pacientes submetidos à angioplastia é bem menor, conforme também foi encontrado no presente estudo.

Laird-Meeter e col. ${ }^{19}$ e Danchin e col. ${ }^{6}$ encontraram diferenças significativas entre os dois procedimentos; já Raft e col. ${ }^{24}$ não dizem, em valores percentuais, qual foi o melhor desempenho de seus pacientes, embora o tamanho de suas amostras fosse pequeno.

Danchin e col. ${ }^{7}$ posteriormente afirmam que com a melhora da técnica da angioplastia ela passa a ser realizada em pacientes com doença da artéria coronária mais extensa.

\subsection{3 - Quanto aos aspectos demográficos}

\section{a) Quanto à idade}

O maior percentual de não-retorno encontra-se na faixa etária dos $60-65$ anos, com $27,2 \%$, seguido da faixa dos $50-59$ anos, com $22,7 \%$, da faixa dos $40-49$ anos com $15,1 \%$ e dos $30-39$ anos com $14,7 \%$.

Vários autores dividiram os pacientes em duas faixas etárias acima e abaixo de 55 anos. No presente caso foi feito divisão semelhante encontrando 411 pacientes abaixo dessa idade. A proporção de não-retorno acima dessa faixa foi de $28,0 \%$, e abaixo igual a $16,3 \%$, diferença esta estatisticamente significante $(p<0,0001)$.

Os pacientes não foram analisados separadamente quanto ao sexo.

Lidlle e col. ${ }^{20}$ encontraram diferença estatisticamente significante quanto ao retorno $\mathrm{em}$ idades superiores e inferiores a 55 anos, sendo que respectivamente não retornaram ao trabalho $28,0 \%$ e $8,0 \%$. 


\section{b) Quanto ao sexo}

Um outro achado, não significativo, foi o retorno ou não ao trabalho, em relação ao sexo do paciente, o que estatisticamente foi demonstrado, existindo total independência entre sexo e retorno ao trabalho ( $>0,05)$ - 26 mulheres em $126(20,6 \%)$ e 114 em 546 homens $(20,9 \%)$ não retornaram.

Tremblay e col. ${ }^{31}$ e Danchin e col. ${ }^{6}$ também não encontraram nenhuma associação significativa entre sexo e retorno ao trabalho.

\subsection{4-Quanto a decisäo pessoal e outras influências}

A Tabela 3 mostra a interferência da decisão pessoal e outras influências no não-retorno ao trabalho.

Tabela 3. Causas de não-retorno ao trabalho segundo os estratos.

\begin{tabular}{lrrrrrr}
\hline & \multicolumn{7}{c}{ Estrato } & & \\
\cline { 2 - 5 } Causa & I & II & III & IV & Total & \multicolumn{1}{c}{$\%$} \\
\hline Aposentou & 11 & 12 & 14 & 26 & 63 & 45,0 \\
Não deixaram & 7 & 12 & 23 & 17 & 59 & 42,1 \\
Renda suficiente & - & - & - & 1 & 1 & 0,7 \\
Firma não aceita & - & - & - & 1 & 1 & 0,7 \\
Outros & 2 & 2 & 7 & 5 & 16 & 11,4 \\
\hline Total & 20 & 26 & 44 & 50 & 140 & 100,0 \\
\hline
\end{tabular}

a) Quanto à decisão pessoal

A decisão de não retornar poderia ter como causa o medo, a insegurança pelo risco de ser acometido de uma sensação de dor, novo infarto ou morte súbita, ou simplesmente o fato, como já foi relatado, de "mudar de vida". Aventou-se a possibilidade do paciente não querer dar detalhes de sua decisăo.

Cay \& Walker ${ }^{3}$ apontam quatro aspectos que têm que ser definidos e delineados:

- O entendimento dos fatos médicos (conhecimento)

- Interpretação subjetiva da doença (crenças)

- Avaliação das implicações pessoais e funcionamento futuro (expectativas)

- Percepção do próprio estado de saúde (percepção da saúde)

Expectativa e percepção da saúde têm-se mostrado intimamente relacionadas com retorno ao trabalho. Insuficiente conhecimento sobre a doença e crenças erradas são associadas com expectativas negativas e sintomas de mal ajustamento e, portanto, falha em reassumir empregos.
Entre as opçoes criadas para o paciente, encontravam-se para respostas as seguintes: a) resolver se aposentar, b) ter medo de sofrer um outro infarto, c) simplesmente não querer retornar, que no cômputo final foram unidos em uma só, aposentou-se.

Resolveu-se tabular junto a estes itens um outro que seria o paciente responder que a pensão ou aposentadoria seriam suficientes para viver. Analisando o resultado, verificou-se que $45,7 \%$ dos casos resultou não-retorno ao trabalho $(45,0 \%$ aposentou e $0,7 \%$, uma paciente do sexo feminino, alegou ter renda suficiente que permitia viver sem trabalhar).

Nenhum outro paciente respondeu que o benefício da previdência social seria suficiente para viver, o que contrasta com Cohen e col. ${ }^{4}$ que relatam grande número de pacientes que poderiam retomar ao trabalho após a cirurgia, mas alguns escolheram tirar vantagens dos benefícios decorrentes da incapacidade, isto porque em alguns países, a legislação protege de tal maneira $o$ indivíduo com problemas de saúde que ocorrem implicações sérias no status ocupacional do paciente. É o caso da Bélgica, conforme relatam Sergeant e col. ${ }^{28}$, onde o incentivo financeiro faz com que os indivíduos permaneçam sem trabalhar após a cirurgia.

\section{b) Quanto à influência de terceiros}

Como segur.do maior resultado, obteve-se de $42,1 \%$ dos pacientes o relato de que o médico e a familia exerceram um fator essencial no nãoretorno ao trabalho.

Frank e col. ${ }^{11}$ são bem incisivos quando afirmam que "a combinação de uma superproteção familiar dizendo ao paciente o que ele não deve fazer, juntamente com o médico falhando em especificar o que ele pode fazer, resulta numa prolongada invalidez e falha em reassumir suas atividades profissionais".

Ressalta-se que os pacientes encaminhados aos hospitais em que a presente pesquisa foi desenvolvida obtiveram alta, estando aptos para retomarem às suas atividades. A partir da alta, esses pacientes passaram a ser acompanhados clinicamente pelos médicos que os encaminharam, já que boa parte eram procedentes do interior e de outros Estados. $\mathrm{O}$ número de pacientes residentes fora do $\mathrm{Mu}$ nicípio de São Paulo, foi o seguinte: $70,0 \%$ no estrato I, $73,1 \%$ no II, 61,3 no III e $52,0 \%$ no IV. A referência quanto ao médico pessoal ou do SUS (Sistema Unificado de Saúde) ter impedido o paciente de retomar, ocorreu em todos os casos de profissionais não pertencentes ao corpo clínico dos hospitais pesquisados. 
David e col. ${ }^{8}$ impressionaram-se com o achado de que $60,6 \%$ dos pacientes atribuíram o seu nãoretorno a conselho médico.

Oberman ${ }^{22}$ vai mais além quando afirma que, a curto prazo, os dados e informações disponiveis não demonstram aumento na produtividade para os pacientes, pois se espera o máximo de benefícios das cirurgias de coronária. Após o procedimento, os médicos que cuidam dos pacientes devem ter um papel mais incisivo no retorno dos pacientes ao trabalho.

Stern e col. ${ }^{29}$, estudando dois grupos de pacientes pós-infarto, encontraram, naqueles envolvidos em um programa educacional intrahospitalar e com apoio ativo de um médico da comunidade, excelentes resultados em termos de retorno ao trabalho e relaçōes sociais.

Tremblay e col. ${ }^{31}$ também acreditam que a atitude positiva da equipe médico-cirúrgica exerce uma influência favorável no retorno do paciente ao trabalho.

Especificamente em relação à família, Ross e col. ${ }^{27}$ são enfáticos ao afirmar que "a maior fonte de apoio vem da esposa(o) e da família, ambas antes e depois da cirurgia". A dependência da familia aumenta à medida que a condição cardíaca deteriora, condição que facilita a influência dela sobre o paciente e reflete no pós-procedimento.

Cay \& Walker ${ }^{3}$ consideram que 0 infarto do miocárdio causa estresse na família e especialmente na esposa(o) que é quem também tem que se ajustar a nova situação. Acrescentam ainda que a ignorância sobre a doença e seu tratamento, o nivel de atividade física segura e o medo de perder uma pessoa amada resulta em superproteção que, por sua vez, leva à frustração e o paciente sente-se irritado e humilhado. Nessas circunstâncias, o retomo ao trabalho toma-se uma ameaça e será postergado o máximo possivel.

Autores como Del Barrio ${ }^{10}$ afirmam que a capacidade de recuperação em pacientes com doenças cardiovasculares é mais alta nos casados.

Ross e col..$^{27}$ também afirmam categoricamente que a maior fonte de apoio vem da(o) esposa(0) e da família, ambas antes e depois da cirurgia, o que demonstra a força dessas pessoas em influenciar decisões do paciente também no que diz respeito ao retorno ao trabalho.

Niles ${ }^{21}$ lembra a importância que tem a atitude do médico quanto à administração do pósoperatório e recomendações ao paciente e a atitude da família, encorajando o paciente, nutrindo um ajustamento com sucesso, evitando-se a proteção que inibe 0 ajustamento em potencial. Desta opinião também compartilham Kushnir e col. ${ }^{18}$.

Vaislic e Passas ${ }^{32}$ enfatizam a necessidade de apoio da equipe na reinserção do paciente no trabalho. Nessa pesquisa, no Canadá, em dois locais diferentes, $47 \%$ e $74 \%$ dos pacientes não retornaram, acreditando esses autores ser decorrente de problemas sociais e econômicos, ressaltando que a equipe teria condições de melhorar estas taxas.

\section{c) Quanto às restriçø̃es do empregador}

Somente um paciente em 672 não retornou ao trabalho devido a não ser aceito pela firma em que trabalhava, em razão dos problemas cardíacos, o que representou apenas $0,7 \%$ do total, consistindo, também, em outro dado isolado da pesquisa.

Em alguns dos trabalhos revisados como o de Symmes e col. ${ }^{30}$ e Oberman e Finclea ${ }^{23}$, esta possibilidade do empregador não aceitar o retorno do trabalhador, também foi citada pelos autores.

\section{d) Quanto a outras circunstâncias}

Na categoria outros, além de incluir os desempregados (7 em 10 não retornaram) foram encontradas diferentes respostas, como por exemplo, um paciente funcionário público federal que se retornasse a trabalhar, seria imediatamente colocado em disponibilidade e ficaria em situação incerta, naquele dado momento político.

Hammerrneister e col. ${ }^{14}$, entre as razões possiveis para o não-retorno, como medo e incentivo econômico para não retornar, cita uma outra hipótese que não foi pensada por nós e que seria a dificuldade encontrada por pacientes mais idosos devido à competição pelo emprego com os mais jovens e com pessoas mais saudáveis. Entretanto, não houve este tipo de caso entre os pacientes $d x$ presente pesquisa.

\subsection{5-Horastrabalhadas antese apóso procedimento}

Analisando-se o número de horas trabalhadas por dia antes e depois do procedimento verifica-se uma variação bastante ampla (Tabela 4).

Tabela 4. Número de horas trabalhadas por dia (HT) antes (A) e depois (B) do procedimento.

\begin{tabular}{lcccccccc}
\hline & HT & -8 & & 8 & & +8 & Total & Total \\
\hline Estrato & A & D & A & D & A & D & A & D \\
\hline I & 14 & 47 & 37 & 56 & 117 & 45 & 168 & 148 \\
II & 29 & 61 & 56 & 49 & 83 & 32 & 168 & 142 \\
III & 34 & 52 & 69 & 45 & 65 & 27 & 168 & 124 \\
IV & 54 & 76 & 45 & 28 & 69 & 14 & 168 & 118 \\
\hline Total & 131236 & 207178 & 334118 & 672 & 532 \\
\hline
\end{tabular}

Obs: Os desempregados estão incluídos na coluna -8A. 
Dos 672 pacientes, $131(19,5 \%)$, incluindo os desempregados, trabalhavam menos de oito horas diárias, $207(30,8 \%)$ trabalhavam exatamente oito horas diárias e $334(49,7 \%)$ tinham uma jornada diária de trabalho que excedia oito horas.

Depois do procedimento, dos 532 pacientes que retornaram ao trabalho, $236(44,4 \%)$ o fizeram com uma carga horária diária menor do que oito horas, $178(33,5 \%)$ passaram a trabalhar oito horas diárias e $118(22,2 \%)$ permaneceram trabalhando por um periodo diário maior do que oito horas.

Resumindo, pode-se afirmar que no quarto mês pós-procedimento não houve ganho, em termos de carga horária trabalhada, pelos pacientes revascularizados que retornaram ao trabalho, visto que $64,7 \%$ dos que trabalhavam mais de oito horas diárias e $14,0 \%$ dos que trabalhavam oito horas reduziram-nas e houve um aumento de $80,1 \%$ naqueles que dispensavam menos de oito horas diárias ao trabalho.

A utilização do teste qui-quadrado demonstra ser altamente significante $(p<0,00001)$ a redução na carga horária diária trabalhada.

Vários autores fizeram essa comparação, entre eles Barnes ${ }^{1}$ que, estudando 350 pacientes, encontrou no pós-procedimento $44 \%$ dos pacientes que diminuiram suas horas trabalhadas, $24 \%$ que permaneceram com a mesma carga horária e $32 \%$ que aumentaram suas atividades. $O$ fato, afirma ele, é que os pacientes após uma grande cirurgia resolvem adotar um novo estilo de vida, o qual não inclui trabalho em tempo integral.

\subsection{6 - Quanto aos antecedentes, hábitos e perfil bioquimico}

Foram selecionados dez fatores para serem pesquisados junto aos pacientes, considerando-se a hipótese de que os mesmos pudessem interferir no retorno ao trabalho. São eles: colesterol, triglicérides, diabetes, ácido úrico, alcoolismo, fumo, obesidade, exercício físico, hipertensão arterial e hereditariedade.

Durante o decorrer da pesquisa, como já ema previsto, surgiram dificuldades na quantificação de alguns deles, como o alcoolismo e o exercício físico, sendo que os mesmos foram abandonados para análise. Optou-se também por não incluir o ácido úrico nessa análise.

Alguns pacientes foram submetidos aos procedimentos sem as dosagens sangüíneas das taxas de colesterol, triglicérides, glicemia e ácido úrico, principalmente naqueles que não vinham sendo seguidos ambulatorialmente.

Resultaram 497 pacientes com todas as dosagens e outros fatores completos, sendo que destes, 11 (22,3\%) não retomaram ao trabalho.
Dos sete fatores, em somente dois deles foi obtida significância estatística em relação à sua influência no não-retorno ao trabalho: hipertensão arterial e tabagismo, mesmo assim não uniformemente em todos os estratos.

A proporção de pacientes hipertensos que não retornaram ao trabalho é significativamente maior que a dos que retomaram nos estratos I $(p<0,000001)$ e II $(p<0,000001)$. Para o fator tabagismo a proporção significante de não - retorno foi encontrada no estrato III $(p<0,05)$.

Entretanto, somente com os dados coletados na presente pesquisa, não é possivel concluir mais profundamente a respeito, desses fatores de influência, merecendo outros estudos.

\subsection{7 - Outros achados de interesse}

a) Dos 140 pacientes que não retornaram ao trabalho original, $20,7 \%(14,4-28,4 \%)$ assumiram uma outra ocupação, encontrando-se em atividade no quarto mês pós-procedimento.

b) Quando perguntados se relacionaram a doença com a ocupação, $31,7 \%(28,2-35,2 \%)$ dos pacientes a confirmaram.

c) Os pacientes de acordo com o seu estado físico e psicológico apresentado após o procedimento, traduzidos pelas atividades físicas, sociais e profissionais, foram classificados em quatro condições: péssimas, regulares, boas e ótimas. O resultado mostrou que somente $5,0 \%$ não cosfrutavam a vida com qualidade, relatando ansiedade, medo da morte, dificuldade de relacionamento, dor e cansaço. Os demais foram classificados em boas $(81,5 \%)$ e ótimas $(13,4 \%)$ condiçðes.

d) Dos 532 pacientes que retornaram ao trabalho original, $96,4 \%$ deles relataram estar sentindose bem.

\section{Comentários Finais}

O não-retorno ao trabalho na presente pesquisa ' mostrou-se percentualmente inferior a maioria dos estudos, visto que $20,8 \%(17,8-23,9 \%)$ dos pacientes não o fizeram, e quando computados aqueles que reiniciaram outras atividades teremos um percentual de somente $16,5 \%(13,7-19,3 \%)$.

A distribuição dos pacientes em quatro estratos ocupacionais, executivo, universitário, técnicos de nível médio e profissionais semi ou não qualificados permitiu verificar um comportamento crescente em relação ao não-retorno ao trabalho, à medida que se analisa do primeiro (I) para o quarto estrato (IV).

Embora somente $31,7 \%(28,2-35,2 \%)$ dos pacientes relacionem a doença com a ocupação, pa- 
rece haver uma intenção em mudar os hábitos de vida pós procedimento terapêutico, independentemente do tratamento utilizado. Mesmo entre os que retomam ao trabalho, em qualquer estrato, há uma tendência à diminuição de horas trabalhadas.

Aqueles submetidos à angioplastia retornam em maior número ao trabalho do que os submetidos à cirurgia.

O sexo não é associado ao retomo ao trabalho, devendo-se direcionar pesquisas que o relacionem com a ocupação, classe social e a morbimortalidade, além do comportamento frente a eles.

Há uma associaçăo entre idade (consideradas duas faixas etárias: acima e abaixo de 55 anos) e não-retorno ao trabalho.

A decisão pessoal e a influência de terceiros são os dois fatores que mais impedem o retorno ao trabalho, sendo que este achado remete a dois problemas básicos que são o conhecimento dos profissionais sobre a doença, a capacidade de recuperação física do paciente e o conhecimento e percepção da familia sobre este aspecto.

Quanto às condições físicas e psicológicas e também aquelas em relação ao trabalho atual pode-se afirmar que os procedimentos terapêuticos obtêm sucesso face ao número absoluto e percentual de pacientes que manifestaram otimismo e segurança após os mesmos.

Finalmente, conclui-se que a distribuição de indivíduos em estratos ocupacionais, como é o caso do presente trabalho, ou em classes sociais, como foram utilizados em outros estudos também citados, pode levar ao conhecimento de importantes aspectos epidemiológicos, que dizem respeito às suas relaçőes com a morbidade, mortalidade, fatores de risco e comportamentos individuais e coletivos. É através deste conhecimento que as açoes preventivas e/ou corretivas poderão ser efetivadas, o que não ocorre quando se ataca somente o convencional, o que é evidenciado por diferentes respostas encontradas nos diferentes estratos ocupacionais ou classes sociais, como no caso das doenças cardiovasculares.

\section{Agradecimentos}

Ao Hospital do Coração da Associação do Sanatório Sírio e ao Instituto Dante Pazzanese de Cardiologia, pelas facilidades oferecidas na coleta de dados.

BITTAR, O.J.N.V. [Retum to work after myocardical revascularization]. Rev. Saúde Pública, 27: 195-203, 1993. Six hundred and seventy-two patients classified in four strata with one hundred sixty eight in each were evaluated during the fourth month after myocardical revascularization, with coronary artery bypass graft and/or internal mammary artery and coronary angioplasty, in order to verify the return or not to work as well as the conditions under which this was done. The four strata constituted by occupational profile were the following: I - entrepreneurs and managers II - professionals with universitary degree III - technicians IV - unskilled and semiskilled professionals. The aim of this research project was to discover how variables like demographics, education, procedure and support after procedure, were related to the return to work. Return to work did not occur in $20.8 \%$ of all cases. The non-return contingent in each of the four strata was the following: $I=11.9 \%: \Pi=$ $15.5 \%$; III $=26.2 \%$ and IV $=29.8 \%$. Among those submitted to angioplasty the proportion of non-return was lower than that of those submitted to surgery.

Keywords: Myocardial revascularization, rehabilitation. Quality of life. Work.

\section{Referénclas Bibliográficas}

1. BARNES, G.K.; RAY, M.J.; OBERMANN, A.; KNOUCHOUKOS, N.T. Changes in working status of patients following coronary bypass surgery. JAMA, 238: 1259 $62,1977$.

2. BOULAY, F.M.; DAVID, P.P; BOURASSA, M.G. Strategies for improving the work status of patients after coronary artery bypass surgery. Circulation, 66: 43-8, 1982.

3. CAY, E.L. \& WALKER, D.D. Psychological factors and retum to work. Eur. Heart J., : (Suppl.): 74-81L, 1988.

4. COHEN, H.A.; SOLNICK, M.; STEPHENSON, A. The financing of coronary antery bypass surgery. Circulation, 66: 49-55, 1982.

5. DANCHIN, N.; DAVID, P.; BOURASSA, M.G.; ROBERT, P.; CHALTMAN, B.R. Factors predicting working status after aortocoronary bypass surgery. Can. Med. Assoc. J., 126: 255-60, 1982 .

6. DANCHIN, N.; JULLIERE, M.; MATHIEU, F.; FAIVRE, G. Reinsertion socio-professionnelle après angioplastie transluminale coronaire. Arch. Mal. Coeur, 77: 993-7, 1984.

7. DANCHIN, N.; DAVID, P.; SELTON-SUTY, C.; VALLANT, G. Retum to work after percutaneous transluminal coronary angioplasty: a continuing problem. Eur. Heart J., 10: 54-7G, 1989.

8. DAVID, P.; TENAILLE, H.; BLAIN, M.; TREMBLAY, H. Etude sur les facteurs de nom retour au travail des cardiaques operes. Un.Med. Can., 105: 1199-205, 1976.

9. DEBUSK, R.F. 20th Bethesda Conference: insurability and employability of the patient with ischemic heart disease. J. Am. Coll. Cardiol., 14: 1008-9, 1989.

10. DEL BARRIO, V. Aspectos psicológicos, su evaluación y tratamiento en enfermos cardiovasculares. Rev. Lat. Cardiol., 9: 310-23, 1988.

11. FRANK, K.A.; HELLER, S.S.; KORNFELD, D.S. A survey of adjustment to cardiac surgery. Arch. Int. Med., 130: 735-8, 1972.

12. GUILLETTE, W.; JUDGE, R.D.; KOEHN, E.; MILLER, J.E.; PALMER, R.K.; TRAMBLAY, J.L.G. Comittee report on economic, administrative and legal factors influencing the insurability and employability of patients with ischemic heart disease. J. Am. Coll. Cardiol, 14: 1010-5, 1989. 
13. GUNDLE, M.J.; REEVES, B.R., JR; TATE, S.; RATT, D. MCLAURIN, L.P. Psychosocial outcome after coronary artery surgery. Am. J. Psychiatry, 137: 1591-94, 1991.

14. HAMMERMEISTER, K.E.; ROUEN, T.A.; ENGLISH, M.T.; DODGE, H.T. Effect of surgical versus medical therapy on retum to work in patients with coronary artery disease. Am. J. Cardiol., 44: 105-11, 1979.

15. HASKELL, W.L.; HASKELL, W.L.; BRACHFELD, N.; BRUCE, R.A. Task force II: determination of occupational working capacity in patients with ischemic heart disease. J. Am. Coll. Cardiol., 14: 1025-34, 1989.'

16. HOLMES, D.R.; VLIETSTRA, R.E.; MOCK, M.B.; SMITH, H.C. Employment and recreation pattems in patients treated by percutaneous transluminal coronary angioplasty: a multicenter study. Am. J. Cardiol., 52: 710-13, 1983.

17. HOLMES, D.R.; VAN RADEN, M.J.; REEDER, G.S.; VLIETSTRA, R.E.; JANG, G.C.; KENT, K.M.; VETROVEC, G.W. Retum to work after coronary angioplasty: a report from the National Heart, Lung, and Blood Institute Percutaneous Transluminal Coronary Angioplasty Registry. Am. J. Cardiol., 53: 41-51, 1984.

18. KUSHNIR, B.; FOX, K.M.; ABER, C.P. Factors influencing the resunnption of work, sexual activity and driving following acute myocardial infarction. Am. Heart $J$. 93: $261-62,1977$.

19. LARD-MEETER, K.; ERDAMAN, R.A.; VAN DONBURG, R.; AZAR, A.J.; FAITER, P.J.; BPSS, E.; HUGENHOLTZ, P.G. Probability of a return to work after either coronary baloon dilatation or coronary bypass surgery. Eur. Heart J., 10: 917-22, 1989.

20. LIDDLE, H.V.; JENSEN, R.; CLAYTON, P.D. The rehabilitation of coronary surgical patients. Ann. Thorac. Surg., 34: 374-82, 1982.

21. NILLES, N.W., II. Retum to work after coronary arery bypass operation. J. Cardiovasc. Surg., 79: 916-21, 1980.
22. OBERMAN, A. Working status of patients following coronary bypass surgery. Am. Heart J, 98: 132-4, 1979.

23. OBERMAN, A. \& FINCLEA, J.F. Retum to work after coronary artery bypass grafting. Ann. Thorac. Surg., 34: $353-59,1982$.

24. RAFT, D.; MCKEE, D.C.; POPIO, K.A.; HAGGERTY, J.J. Life adaptation after percutaneous transluminal coronary angioplasty and coronary artery bypass grafting. Am. J. Cardiol., 56: 395-8, 1985.

25. RIMM, A.A.; BARBORIAK, J.J.; ANDERSON, A.J.; SIMON, J.S. Changes in occupation after aorta/coronary vein-bypass operation. JAMA, 236: 361-4, 1976.

26. RODIS, S.M. Work status after artery bypass operation. $J$. Cardiovasc. Surg., 26: 228-30, 1985.

27. ROSS, J.K.; DIWELL, R.E.; MARSH, J.I.; BARKER, D.J.P. Wessex cardiac surgery follow-up surgery: the quality of life after operation. Thorax, 33: 3-9, 1978.

28. SERGEANT, P.; LESFFRE, E.; FLAMENG, W.; SUY, R. How predictable is the postoperative workresumption after aortocoronary bypass surgery. Acla Cardiol., 41: 41-52, 1986

29. STERN, MJ.; PASCALE, L.; ACKERMAN, A. Life adjustment post-myocardial infarction: determining predictive variables. Arch.Intern. Med., 137: 1680-5, 1977.

30. SYMMES, J.C.; LENKEI, S.C.M.; BERMAN, N.D. Influence of aorto-coronary bypass surgery on employment. Can. Med. Assoc., 118: 268-70, 1978.

31. TREMBLAY, G.; BIRON, P.; PELLETIER, L.C.; COSSETTE, R.; DONTIGNY, L; PROULX, A. Retour au travail apres pontage aorto-coronarien. Un. Med. Can., 105: 1206-8, 1976.

32. VAISLIC, C.L. \& PASSAS, H. Reprise précoce du travail aprés chirurgie arteriele et contrôle strict des invalides. Phlébologie, 42: 323-26, 1989.

Recebido para publicaçdo em 26.11.1992 Aprovado para publicaçđo em 31.3 .1993 